\title{
Road Navigation System Monitoring using a Pseudorange Snapshot Test
}

\author{
Clément Fouque* Philippe Bonnifait* \\ * Heudiasyc UMR CNRS 6599, \\ Université de Technologie de Compiègne \\ BP20529 , 60205 Compiègne Cedex, France \\ clement.fouque@hds.utc.fr, philippe.bonnifait@hds.utc.fr
}

\begin{abstract}
Global Navigation Satellite Systems (GNSS) are often used to localize a receiver with respect to a given map. This association problem, also known as map-matching, is usually addressed using estimated positions computed by the GNSS receiver. This paper focus on the use of GNSS raw data (L1 pseudo-measurements) and cartographic data provided by a digital road network. Using the positioning residuals of map-aided GNSS, a new method for tackling the underlying problem of the road selection is proposed. This paper show that this approach is also well adapted to integrity problem of map-matching, since a consistency test is derived. Experimental results illustrate the performance of this method with different maps.
\end{abstract}

Keywords: Positioning systems; GNSS; GIS; Data fusion; Robotics systems integrity.

\section{INTRODUCTION}

Global Navigation Satellite Systems (GNSS) including GPS, Glonass and Galileo, along with their Satellite-Based Augmentation Systems (SBAS), are very promising affordable technologies for many applications. Very often, absolute positioning is useful when associated with a geographical database that contains a priori information. A good example is a GPS-based navigation system that uses road network information for route planning or contextual information retrieval, such as road curvature estimation, speed limits, or points of interest if stored. All this data is spatially-indexed and stored in different layers managed by Geographic Information Systems (GIS). A GIS alows accessing, modifying, enhancing and displaying Geographical Information (GI). Moreover, as the amount of GI increases rapidly, available information is highly diversified. In addition to the road network, information like terrain elevation, natural landmarks, buildings description ..., is now available. Therefore, this a priori knowledge of the environment can be used in the localisation process itself for precise positioning in urban areas (Jabbour et al. [2006], Meizel et al. [2005]).

The problem of localizing a receiver with respect to a map is known as map-matching. Usually this problem is tackled using GNSS fixes provided by a receiver, i.e. position solutions computed using pseudo-ranges and ephemeris data. The GNSS data is often fused with dead-reckoning in order to improve positioning availability and accuracy (Lahrech et al. [2005], Meizel et al. [2005], Laneurit et al. [2005]). The main drawback of this approach is the need of DR sensors and at least four visible satellites, conditions that are rarely satisfied in urban canyons (Georgiev and Allen [2004]). Moreover, integrity monitoring is difficult to assess in such cases, as the pseudo-range measurements can suffer from multipath. An alternative is a tightly-coupled approach including map information in fix computation. This approach, which also allows mixing GPS, Glonass or Galileo pseudo-ranges is the one addressed in this paper particulary for integrity purposes, since multipath mitigation (Betaille et al. [2003]) can be tackled. Monitoring the positioning integrity is indeed crucial for many land vehicle applications (Santa et al. [2006]). As a matter of fact, Required Navigation Performance (RNP) are under development in order to standardize road-transport application integrity (Feng and Ochieng [2007]).

This paper investigates the use of a road network map provided by cartographers. This information describes the center-lines of carriageways in a $2 \mathrm{D}$ representation. The main difficulty is how-to use such information in GNSS computation. To this end, a navigation frame, where satellite positions are known, is built. Firstly, supposing that the road is known, the way to compute a location is recalled (Fouque and Bonnifait [2007]). Then, a strategy for selecting the most likely road by using the residuals of the computation is presented. These residuals also allow an integrity test to be performed. Experimental results carried out with our experimental car show the performance of the approach: With 3 visible satellites, the map-matched location can be computed. And, with 4 visible satellites at least, an integrity test can be performed to quantify the confidence in this map-matched location.

The paper is organized as follows. Section 2 provides a reminder of map-aided GPS positioning methods. Using these methods, a candidate segments extraction algorithm is presented in Section 3. Then, an integrity analysis using a choosen probability of false detection is proposed in Section 4 for selecting the most likely road segment. Section 5 presents real experimental results carried out using the proposed road selection algorithm and the integrity test derived from the new map-aided GNSS positioning. 


\section{GIS DATA IN GNSS COMPUTATION}

Two methods for including geographical information in GNSS fix computation are presented in thi section. The use of digital road maps illustrates this purpose. In the following, the assumption that vehicles evolves on a known road segment is made.

For GNSS computation, pseudo-range positioning is used:

$$
\rho^{i}=R^{i}+c \cdot d t_{u}+\delta_{\rho}^{i}, \quad \forall i=1, \ldots, n
$$

where $\rho$ is the measured pseudo-range, $R$ the receiver to satellite $(\mathrm{SV})$ geometrical distance, and $\delta_{\rho}$ the ranging errors. The corresponding observation model (2) is then solved using Least Squares (LS), see (Fouque and Bonnifait [2007], Kaplan [1996]) for details.

$$
\rho=h\left(x_{u}, y_{u}, z_{u}, d t_{u}\right)
$$

\subsection{Road maps}

A digital road map is a database that contains a vectorial description of the road network. Roads are described in a discrete fashion by their center-lines. Data associated to a road are classified into three groups:

- Geographical: A segment set describing the road geometry.

- Topological: Description of connectivity between road segments.

- Semantic: Road name, speed limit, etc...

Digital road maps can achieve a metrical precision, which is sufficient for many navigation tasks including route planning. To overcome memory and real-time constraints, roads around the vehicle position are extracted (typically $1 \mathrm{Km}^{2}$ ) and cache is stored in memory.

\subsection{Working Frame}

As GPS ephemeris data are provided in WGS84 Cartesian frame, and road maps in various frame depending on the provider, a common working frame is need to compute a valid tightly-coupled GNSS/map-matching positioning solution. Here, roads are depicted as part of Earth surface: map nodes and shape points are only described by their longitude and latitude, assuming their elevation equals 0 .

Using map geographical data, it useful to determine a tridimensional local frame (denoted $l o c$ ) such that its plane $(O, i, j)$ is tangential to the WGS84 earth reference ellipsoid. First, the map points coordinates are converted from the geodetic WGS84 to the Cartesian WGS84 frame. The origin $O$ is chosen to correspond to the origin node of a road close to the estimated position. The $i$ axis was made to correspond to the first segment of this road and the $(O, i, j)$ plane is characterized by a geometry point of any other nearby road, provided that this point is not situated on the $i$ axis. Finally, the $k$ axis is chosen such that the local frame is right-handed.

A homogeneous transform ${ }^{l o c} T_{W G S 84}$ is then computed. It contains the rotation and translation terms needed to apply the transform. Using ${ }^{l o c} T_{W G S 84}$, the satellite coordinates and the map cache, geometry points can be converted in the working frame.
It should be noted that the working frame is temporary, typically for a road cache (Bonnifait et al. [2007]), and valid only for small regions (limited to several kilometers), which addresses the meridian convergence problem.

\subsection{Plane Constraint for Computation}

Let suppose that the correct road segment has been selected from the road points given by the GIS. The constraint defined by this selected segment is part of a vertical plane (in the working frame), since the elevation of the map is unknown. In practice, the whole plane is considered and the matched position $\left(x_{m}, y_{m}\right)$ is checked to be within segment bounds.

Taking $M\left(m_{1}, m_{2}\right)$ and $N\left(n_{1}, n_{2}\right)$ as the extremities of the segment, its equation is:

$$
\left\{\begin{array}{l}
x=m_{1}+\left(n_{1}-m_{1}\right) \cdot l \\
y=m_{2}+\left(n_{2}-m_{2}\right) \cdot l \\
z \quad \text { free }
\end{array} \quad \forall l \epsilon[0,1]\right.
$$

The geometrical equation Eq.3 means that only the computation along $(x, y)$ is constrained. For the computation, the segment constraint is relaxed, and a straight line is used:

$$
y=f_{1}(x) \quad \text { if } n_{1} \neq m_{1}
$$

\subsection{First method: Unknown Elimination}

This method was proposed in (Cui and Ge [2003]). The idea is to eliminate a variable using the constraint equation. The geometrical distance between the receiver and $\mathrm{SV}^{i}$ can be rewritten as

$$
R^{i}=\sqrt{\left(x-x^{i}\left(t_{e}^{i}\right)\right)^{2}+\left(f_{1}(x)-y^{i}\left(t_{e}^{i}\right)\right)^{2}+\left(z-z^{i}\left(t_{e}^{i}\right)\right)^{2}}
$$

Thus, introducing Eq.4 in Eq.2 gives a new non-linear system:

$$
\rho_{c}^{i}=g^{i}\left(x_{u}, z_{u}, d t_{u}\right), \forall i=1, \cdots, n
$$

The dimension of the problem has now reduced, and the minimum number of SVs necessary for computing the positioning solution is 3 . Since the constraint is strong, the computed position belongs to the constraint plane. It should be noted that its projection onto the map plane can be outside the segment.

\subsection{Second method: Plane Fusion}

This method was proposed by S. Syed and M.E. Cannon in (Syed and Cannon [2005]). Using the segment parameters, a new observable is built. Therefore, it becomes possible to add a new equation to the observation model defined by Eq.2. So, the pseudo-measurement $\rho_{c}^{n+1}$ extracted from the GIS data and its associated observation equation is defined as:

$$
\begin{aligned}
\left(n_{1}-m_{1}\right) m_{2}+\left(m_{2}-n_{2}\right) m_{1} & =\left(n_{1}-m_{1}\right) y+\left(m_{2}-n_{2}\right) x \\
\rho_{c}^{n+1} & =h^{n+1}\left(x_{u}, y_{u}, z_{u}, d t_{u}\right)
\end{aligned}
$$

Thus, an extended observation model can be written as:

$$
\tilde{\rho}_{c}=\tilde{h}(s)
$$

With $s$ being the state vector such that $s=\left[x_{u}, y_{u}, z_{u}, d t_{u}\right]^{t}$ and $\tilde{\rho}_{c}=\left[\rho_{c}, \rho_{c}^{n+1}\right]^{t}$. 
Using this additional measurement and at least three SVs, the positioning solution can be computed. In contrast to the unknown elimination method, the computed solution is not located within the constraint plane defined by the road segment.

Moreover, GIS data can be balanced according to a quality indicator in a Weigthed LS solver Let denote $W$ the weighting factor for the pseudo-ranges. After convergence, the matched approximate solution $\hat{s}_{m}$ can be expressed by:

$$
\hat{s}_{m}=\tilde{H}^{+}\left(\hat{s}_{m}\right) \cdot\left(\rho_{c}-\tilde{h}\left(\hat{s}_{m}\right)\right)
$$

With

$$
\begin{gathered}
\tilde{H}^{+}\left(\hat{s}_{m}\right)=\left[\tilde{H}^{t}\left(\hat{s}_{m}\right) \tilde{W}^{-1} \tilde{H}\left(\hat{s}_{m}\right)\right]^{-1} \tilde{H}^{t}\left(\hat{s}_{m}\right) \tilde{W}^{-1} \\
\tilde{H}\left(\hat{s}_{m}\right)=\left.\frac{\partial \tilde{h}}{\partial s}\right|_{\hat{s}_{m}} \quad \tilde{W}=\left[\begin{array}{cc}
W & 0 \\
0 & \sigma_{G I S}^{2}
\end{array}\right]
\end{gathered}
$$

Where $\sigma_{G I S}$ is the map quality.

Introduction of road segment information into the positioning solution computation have been presented. Next, the underlying problem of segment choice is tackled by a road selection algorithm.

\section{EXTRACTION OF CANDIDATE SEGMENTS}

The road selection comprises three steps. First, candidate segments are extracted from the road cache according to GNSS observations. Then, candidate segments that fail to match a consistency criterion are eliminated, and finally the most likely segment is selected.

This first step of the road selection method involves the extraction of candidate segments from current road cache. For each segment, a tightly-coupled positioning solution is computed using the unknown elimination method in order to determine the corresponding matched point. A non-linear equation system like Eq.6 is then solved for each road segment in the cache using LS. As the provided solution is constrained to a vertical plane defined by the road segment (see Section 2.4), any segment is a candidate if it satisfies the two following conditions:

(1) The projection of the fix onto the reference plane $(O, i, j)$ belongs to the segment. As the segment shape points are known, a simple test can be performed involving the projection of the fix onto the reference plane $(O, i, j)$. Let define the segment extremities as $M$ and $N$. Using a scalar product, the projection $P$ of the computed fix onto $(O, i, j)$ belongs to the road segment if:

$$
M P \cdot N P \leq 1
$$

(2) The altitude of the matched fix should be close to 0 in the working frame. Therefore, a threshold (denoted $T h_{\text {alt }}$ ) is applied in order to eliminate outlying solutions:

$$
z<T h_{\text {alt }}
$$

where $z$ defines the altitude of the matched point in the working frame.

Using the conditions defined by Eq.11 and Eq.12, a set of candidate segments is obtained. This set can either be empty, or not contain the true road segment if, for instance, a wrong cache is used.

\section{INTEGRITY OF MAP-MATCHING}

Software integrity methods suppose that there is a redundancy in the measurements (Belabbas and Gass [2005], Sturza and Brown [1990]). Using GIS data in the GNSS computation reduces the number of satellites required in line of sight. Either there are 3 unknowns and $n$ measurements (see Eq.6), or there are 4 unknowns and $n+1$ measurements (see Eq.8). So, with $n$ satellites, the degree of redundancy is $n-3$. Therefore 4 visible satellites at least are assumed for monitoring integrity.

In this section, the plane fusion method is used for integrity analysis since it allows map veracity to be weighted. The considered integrity test relies on a consistency check of residuals: assuming that inconsistencies can result only from wrong matches (and not from GNSS faults), this test allows the selection of the most likely segment and integrity monitoring of a road selection process.

\subsection{Positioning residuals}

In order to determine whether or not a segment is consistent, the LS positioning residuals are used. As this solver is iterative, the residuals vector is computed after convergence of the solution. Let rewrite Eq.8 with the residuals vector $\varepsilon$ :

$$
\varepsilon=\tilde{\rho}_{c}-\tilde{h}(\hat{s})
$$

$\hat{s}$ is the last solver estimated solution. Given the linearization point $\hat{s}_{0}$ and the variation vector $d s$, the positioning residuals vector $\varepsilon$ is:

$$
\varepsilon=\tilde{\rho}_{c}-\tilde{h}\left(\hat{s}_{0}+d s\right)
$$

Where $d s$ is obtained at the last step of the solver:

$$
d s=\tilde{H}^{+}\left(\hat{s}_{0}\right) \cdot\left(\tilde{\rho}_{c}-\tilde{h}\left(\hat{s}_{0}\right)\right)
$$

Consequently, using a first-order linearization, the residuals vector is now:

$$
\varepsilon=\left(I_{n+1}-\tilde{H}\left(\hat{s}_{0}\right) \cdot \tilde{H}^{+}\left(\hat{s}_{0}\right)\right)\left(\tilde{\rho}_{c}-\tilde{h}\left(\hat{s}_{0}\right)\right)
$$

It should be remarked that this is a weighted residuals vector.

\subsection{Elimination of inconsistent segments}

For each candidate segment, the Euclidean norm $\|\varepsilon\|$ of the weighted positioning residuals is computed from Eq.16 using the last iteration results of the LS solver. In order to eliminate the inconsistent segments, a $\chi^{2}$-test is performed for every candidate segments under Gaussian assumptions (Walter and Enge [1995]). If the square norm of the residuals exceeds a certain threshold $T h_{\text {con }}$ then the current candidate segment is eliminated. Assuming the candidate segment to be faulty and good GPS measurements, this strategy can be interpreted as degenerated RAIM (Receiver Autonomous Integrity Monitoring) fault isolation.

The problem is now to define the threshold $T h_{c o n}$. In our case, we consider that the pseudo-range noise can be different for each satellite in view of the SBAS correction, and 


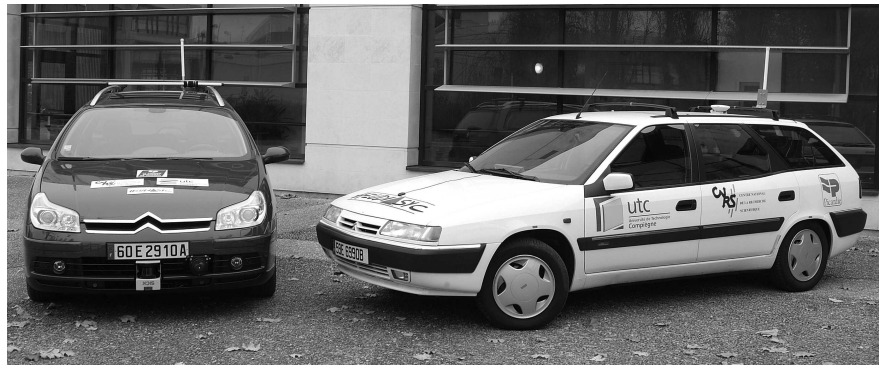

Fig. 1. HeuDiaSyC experimental vehicles: Carmen (left) and Strada (right)

also that the map error is known. In (Belabbas and Gass [2005]), the authors propose a Cholesky decomposition of $\tilde{W}$ (see Eq.10):

$$
\tilde{W}=A \cdot A^{t}
$$

Let consider the new normalized positioning residuals $\varepsilon^{\prime}$ :

$$
\varepsilon^{\prime}=A^{-1} \cdot \varepsilon
$$

A segment is therefore consistent if

$$
\left\|\varepsilon^{\prime}\right\|^{2}<T h_{c o n}
$$

where $T h_{c o n}$ is an adaptativ threshold computed using the inverse of a $\chi^{2}$ cumulative distribution function with $(n-3)$ degrees of freedom and a given $P_{f a}$ (probability of false alarm) depending on the application.

This stage might yield no segment at all (if the vehicle is off-road, for instance), or several segments in case of ambiguous situation.

\subsection{Segment selection}

If not any consistent segment is provided, map data can not be fused with GNSS data because the vehicle is probably not located on a road within the cache. This fact is useful for detecting cache management troubles. Otherwise, if there are several consistent segments, either a most likely segment can be chosen, or multi-hypothesis matching can be carryied out, involving Interacting Multiple Models (IMM) at each junction (Cui and Ge [2003]).

In order to select the most likely segment from a set of consistent segments, a simple strategy is to choose the one with the lowest positioning residuals norm. Let us study this strategy using real data.

\section{EXPERIMENTAL RESULTS}

\subsection{Methodology}

Experiments were carried out in two stages duing July 2006. The first stage was the data recording, followed by data processing. Data were recorded using our Laboratory's experimental vehicle Strada (Fig.1) and a Trimble 5700 GPS receiver in stand-alone mode. These records were made on roads near the lab which are well identified in the geographical database. SV measurements were recorded using Rinex 2.10 observation file format and the corresponding navigation file was used. Using the Rinex files recorded by the receiver, a DGPS PPK reference

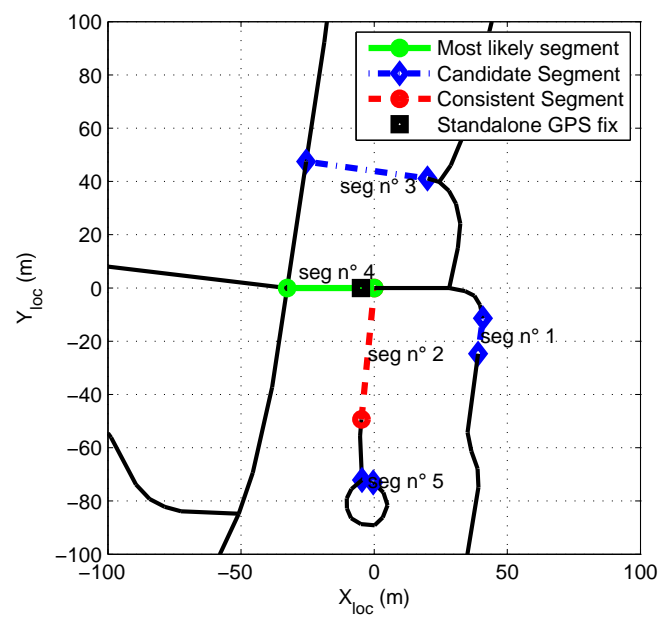

Fig. 2. Results of the road selection algorithm on a static point

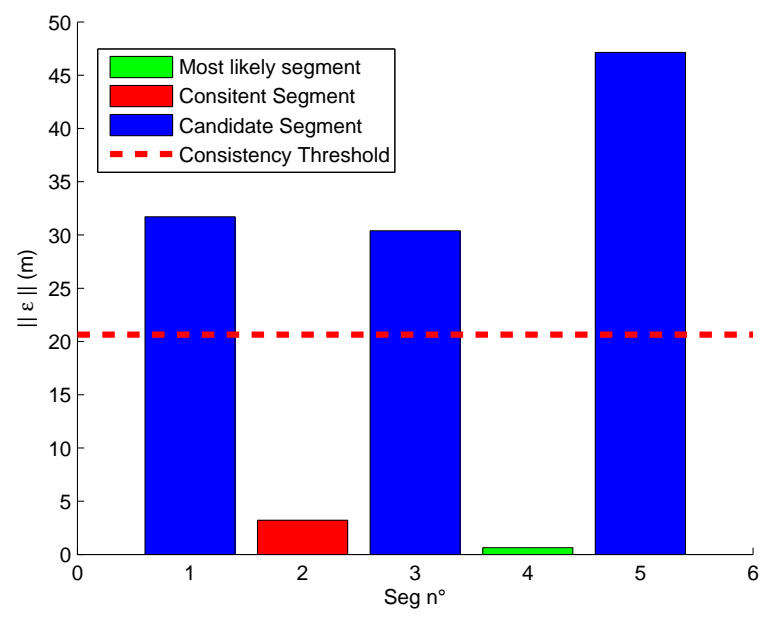

Fig. 3. Residual for each candidate segment

trajectory was computed using Trimble Total Control software and data from several reference stations (French Orpheon network).

For every experiments, a fixed road cache was extracted from a GIS database provided by a cartographer. The road cache was extracted around the lab, with a diameter of about $600 \mathrm{~m}$.

In these experiments, the weight matrix $W$ and the extended weight matrix $\tilde{W}$ were set to identity. So, the standard deviation $\sigma_{i}$ of the pseudo-ranges and the standard deviation $\sigma_{\text {GIS }}$ of the map were assumed to be equal.

$$
\sigma_{i}=\sigma_{G I S}=\sigma_{U E R E}
$$

The integrity threshold $T h_{\text {con }}$ was computed using a $P_{f a}$ such that one false alarm per hour is permitted, which seems acceptable for many land vehicle applications $\left(P_{f a}=2.75 \cdot 10^{-4}\right)$. Given this simplification, the integrity test (Eq.19) is reduced to:

$$
\left\|\varepsilon^{\prime}\right\|<\sigma_{U E R E} \cdot \sqrt{T h_{c o n}}
$$



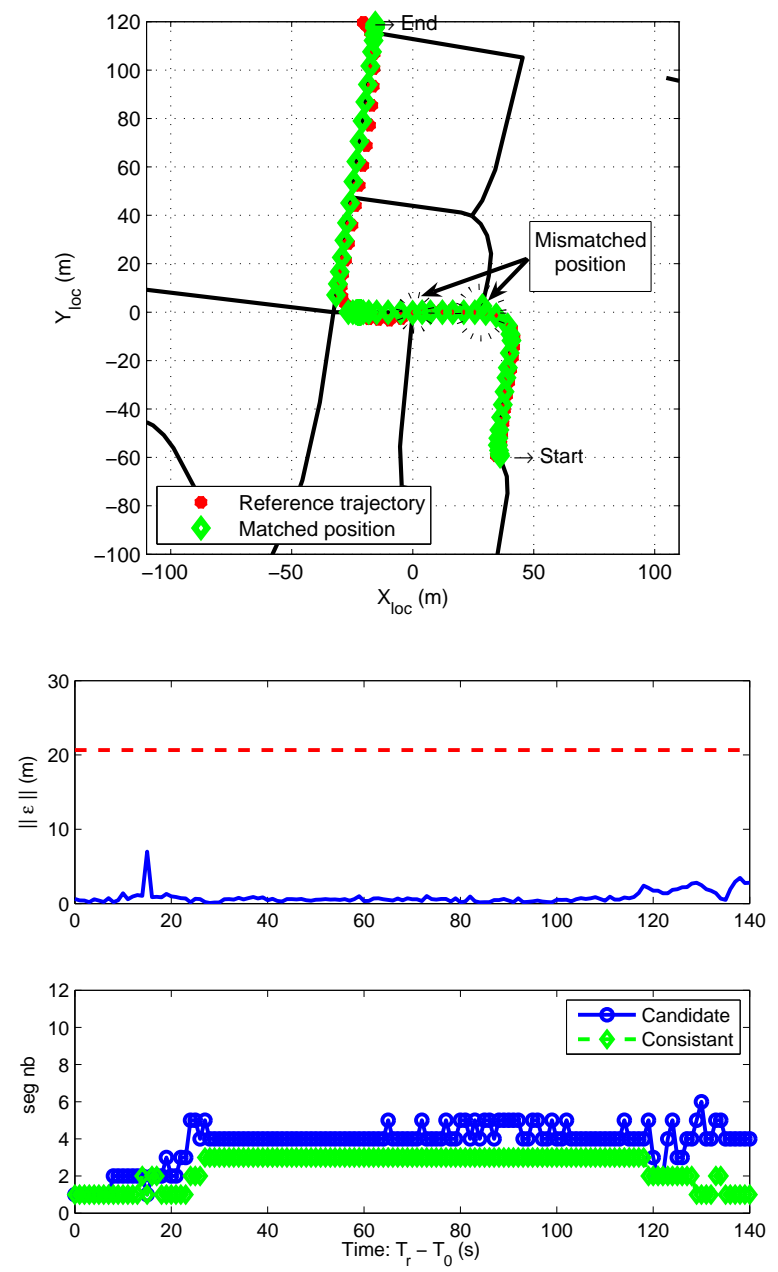

Fig. 4. Results of the matched positioning computation with aligned map

With $\sigma_{U E R E}$ (User-Equivalent Range Errors) the onesigma effective pseudorange errors $(12.5 \mathrm{~m}$ without SBAS corrections).

\subsection{Road selection using integrity}

In this section, the road selection algorithm and the tightly-coupled GNSS-Map fusion are analyzed using a single fix. The candidate segment extraction and the integrity test are computed for the same position, and the results are compared to the standalone GPS fix. For this test, map biases were removed and all the visible satellites were used. For simplicity, the local frame was made to correspond to the nearest road junction. The $(O, i)$ axle coincides with one of the segments composing this road junction. In reality, this segment is not East-oriented.

The results of road selection are shown in Fig.2 and the results of the integrity test are shown in Fig.3. One can see here that the candidate extraction stage provides 5 candidates segments. For those segments, the integrity test is applied to extract the consistent segment.

The integrity test of Section 4 yields only two consistent segments (cf Fig.3). Considering the corresponding standalone GPS fix, one can see that the vehicle is located
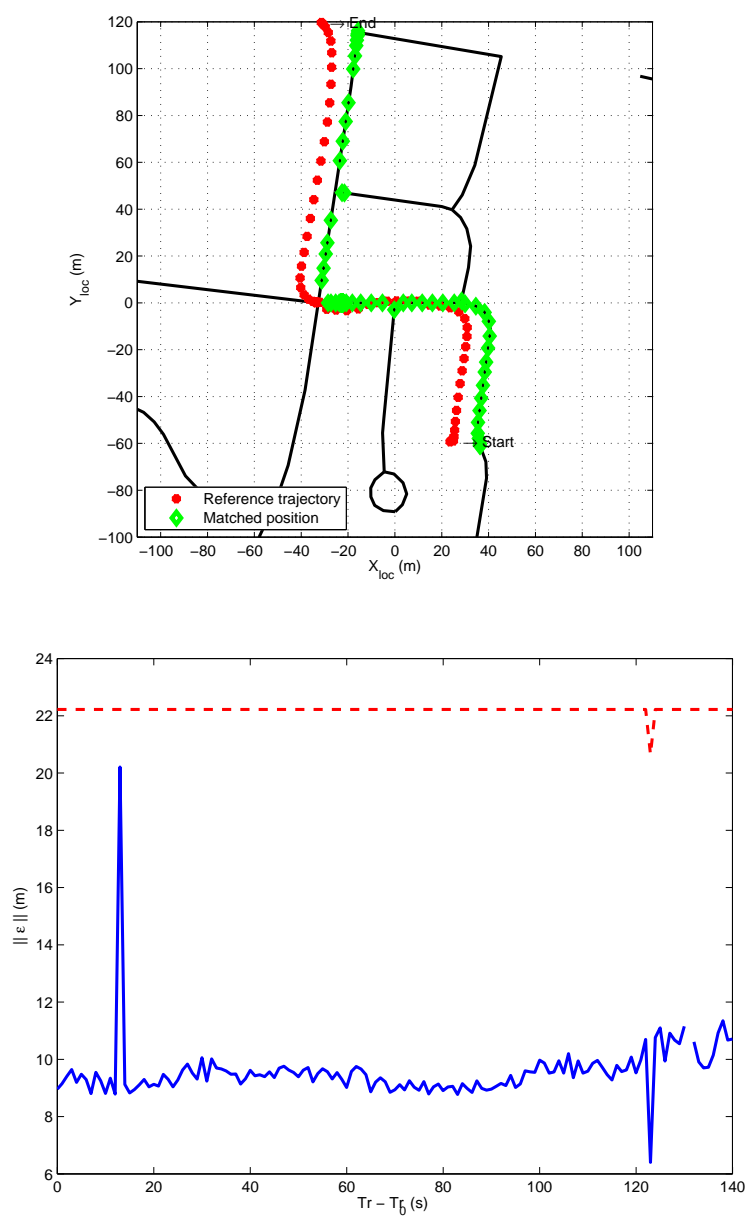

Fig. 5. Residuals of the matched positioning computation with real map

near a road junction, which is an ambiguous situation as the two consistent segments are those composing the road junction.

\subsection{Full road test with an aligned map}

In this section, computation results for matched positions are presented. The behavior of our method, during a dynamic test using all the visible satellites ( 5 in this experiment) and aligned map data, is reported. Please note that, in these tests, the vehicle stops at the 4-way junction during 70 seconds (between the 40th and the 110 th second).

During this test, two mismatched positions occurrs when the two first road junction are encountered, and another mismatch appears between the starting point and the first junction, where the road is depicted by numerous small segments (see Fig.4). The conclusion is that in this ambiguous situation an incorrect road segment is chosen because the vehicle's true segment failed to feature among the candidates. Looking at the norm of the positioning residuals, one can notice a peak around second 17, after the start. This peak value corresponds effectively to the mismatch. Unfortunately, the norm of the residuals falls below the integrity threshold, and so the mismatch is not 
detected. Throughout the test, one can remark that the value of the residuals norm remains low, that is to say around 1 meter up until the 120th second, and around $3 \mathrm{~m}$ afterwards, which is very good.

Regarding the selection of consistent segments, one can see that there is always at least one consistent road. On average, 2 or 3 are declared consistent, which indicates that the method hesitates often. This conclusion suggests either that the data-association strategy is over-cautious, or that the selection is difficult to assess.

\subsection{Full road test with a real map}

In practice, road maps are often biased, and usually this bias is less than 10-15 meters.

One can see on Fig. 5, despite the map bias, that the mapmatched locations are mainly correct. Several mismatches $(\sim 7.8 \%)$ appear close to the different road junctions that are naturally ambiguous area. When looking at the positioning residuals norm, one can notice several interesting phenomena. The first peak value corresponds to the first one observed in Section 5.3: a mismatch at the first junction. Another interesting point is the gap in the residuals. At time $t \sim 130$ no segment passed the integrity test and no position was computed. Finally, one can see that the average level of the positioning residuals norm is about 9 meters. It actually corresponds to the norm of the bias of the map.

\section{CONCLUSION AND FUTURE WORK}

In this paper, a method to select the current road evolution segment from a digital road network have been described. To achive this selection, a method based on a thightly coupled fusion of GNSS raw measurements (L1 pseudoranges) and geographical data extract from the digital road map has been proposed. Using a simple snapshot integrity test on map-aided positioning residuals, this method is able to provide a set of confident segments to the enduser. The use of SBAS information has also been taken into account, as the integrity test is applied on weigthed positioning residuals. To illustrate this proposal, a simple strategy provides the most likely evolution segment. The results that have been obtained in stand-alone GPS are very encouraging, since the method is able to retrieve the correct segment if the map is unbiased (only one incorrect match occurred using a map provided by a cartographer). When using a real biased map, the performance unsurprisingly decreases and the method outputs several false matches. In this case, our integrity test inform the end-uer since the residuals norm indicates a significant map bias.

The perspectives of this research concern the use of multihypothesis dynamic state observers (based on Kalman filters) to exploit road connexion, particularly when approaching junctions. Moreover, the use of WAAS or EGNOS corrections will be tested as a mean of reliability improvement.

\section{ACKNOWLEDGEMENTS}

This research was carried out within the framework of the European FP6 Integrated Project CVIS ( $\left.n^{\circ} 027293\right)$, launched in February 2006 for a duration of four years.

\section{REFERENCES}

B. Belabbas and F. Gass. Raim algorithms analysis for a combined gps/galileo constellation. In ION GNSS 2005, Los Angeles, CA, USA, 2005.

D. Betaille, J. Maenpa, H.J. Euler, and P.A. Cross. A new approach to gps phase multipath mitigation. In ION National Technical meeting - NTM2003, pages 243-253, Anaheim, CA, USA, 2003.

Ph. Bonnifait, M. Jabbour, and G. Dherbomez. Realtime implementation of a gis-based localization system for intelligent vehicles. EURASIP Journal on Embedded Systems, vol. 2007:12-24, June 2007. DOI $10.1155 / 2007 / 39350$.

Y.J. Cui and S.S. Ge. Autonomous vehicle positioning with gps in urban canyon environments. IEEE Trans. on Rob. and Aut., 19:15-25, 2003.

S. Feng and W.Y Ochieng. Integrity of navigation system for road transport. In Proceedings of the 14th World Congress on Intelligent Transport Systems, Beijing, Octobre 2007.

C. Fouque and P. Bonnifait. Vehicle localization in urban canyons using geo-referenced data and few gnss satellites. In IFAC Symposium on Intelligent Autonomous Vehicle - IAV200\%, Toulouse, France, Sept 2007.

A. Georgiev and P. K. Allen. Localization methods for a mobile robot in urban environments. IEEE Trans. on Rob. and Aut., 20:851-864, 2004.

M. Jabbour, Ph. Bonnifait, and V. Cherfaoui. Management of landmarks in a gis for an enhanced localisation in urban areas. In IV2006 IEEE In. Veh. Symp., Tokyo, Japan, June 2006.

E.D. Kaplan. Understanding GPS: principles and applications. Artech House, 1996.

A. Lahrech, C. Boucher, and J-C Noyer. Accurate vehicle positioning in urban areas. In The 31st Annual Conference of the IEEE Industrial Electronics Society IECON 2005, Raleigh, NC, USA, 2005.

J. Laneurit, R. Chapuis, and Chausse F. Accurate vehicle positioning on a numerical map. Int. J. of Control, Automation and System, 3(1):15-31, 2005.

D. Meizel, S. Renault, and A. Le Meur. Gps/gis localization for management of vision referenced navigation in urban environments. In IEEE Conference on Intelligent Transportation Systems -ITSC2005, Vienna, Austria, 2005.

J. Santa, B. Ubeda, R. Toledo, and A.F.G. Skarmeta. Monitoring the position integrity in road transport localization based services. In IEEE Vehicular Technology Conference - VTC2006, pages 1-5, Montreal, QC, Canada, 2006.

M.A. Sturza and A.K. Brown. Comparison of fixed and variable threshold raim algorithms. pages 437-442, NAVSYS Corp, Monument, United States, 1990.

S. Syed and M.E. Cannon. Map-aided gps navigation. GPS World, 16(11):39-44, 2005.

T. Walter and P. Enge. Weighted raim for precision approach. In ION GPS 1995, Palm Springs, CA, USA, 1995. 